\title{
Magnetic Resonance Imaging of Rectal Cancer
}

\author{
Mai-Lan Ho, M.D., ${ }^{1}$ Judy Liu, M.D., ${ }^{2}$ and Vamsidhar Narra, M.D. ${ }^{2}$
}

\section{ABSTRACT}

Magnetic resonance imaging (MRI)is a useful modality for the evaluation of rectal cancer, providing superior anatomic/pathologic visualization when compared with endorectal ultrasound (EUS) and computed tomography (CT). Preoperative MRI is useful for tissue characterization and tumor staging, which determines the surgical approach and need for neoadjuvant/adjuvant therapy. Important prognostic factors include the circumferential resection margin $(\mathrm{CRM}), \mathrm{T}$ and $\mathrm{N}$ stages, and extent of local invasion. Postoperative MRI to assess the extent of tumor recurrence enables early resection, which can greatly prolong survival. MRI criteria for local recurrence include T2 hyperintensity, early dynamic rim enhancement, and nodular morphology. Future research in MRI of rectal cancer is geared toward developing optimal imaging techniques including highresolution MRI, whole-body scans, and parallel imaging; imaging of lymph nodes by MR lymphography; and response to therapy using diffusion/perfusion-weighted MR and functional imaging.

KEYWORDS: Magnetic resonance, rectal cancer, preoperative, postoperative, recurrence

Objectives: On completion of this article, the reader should be able to summarize the utility of magnetic resonance imaging (MRI) in the pre- and postoperative assessment of rectal cancer.

Colorectal cancer is the most common form of gastrointestinal tract cancer, and a leading cause of morbidity and mortality in developed countries. Rectal carcinoma represents 40 to $50 \%$ of colorectal cancers, with a significant risk of local recurrence and distant metastases. Risk factors include a high-fat, low-fiber diet; age greater than 50 years; male gender; personal or family history; predisposing conditions such as hereditary nonpolyposis colorectal cancer (HNPCC or Lynch); familial adenomatous polyposis (FAP), Gardner, Turcot, juvenile polyposis, and Peutz-Jeghers syndromes; and inflammatory bowel disease.

\footnotetext{
${ }^{1}$ Washington University School of Medicine, St. Louis, Missouri;

${ }^{2}$ Abdominal Radiology Section, Mallinckrodt Institute of Radiology, Washington University School of Medicine, St. Louis, Missouri.

Address for correspondence and reprint requests: Vamsidhar Narra, M.D., Mallinckrodt Institute of Radiology, Washington University School of Medicine, Campus Box 8131, St. Louis, MO 63110 (e-mail: narrav@wustl.edu).
}

Prognosis in rectal cancer is also affected by histologic grade, with less differentiated tumors being more aggressive; and by histologic type. The majority (98\%) of rectal cancers are adenocarcinomas, which follow the adenoma-carcinoma sequence of pathogenesis. Particularly aggressive forms include the mucinous (colloid) and signet ring subtypes. Neuroendocrine carcinomas tend to have a poorer prognosis than adenocarcinomas, particularly the pure neuroendocrine and small-cell forms. Carcinoid tumors are the exception, being slow-growing and easily resectable, and indolent even when metastatic. Scirrhous carcinoma is a diffusely infiltrating anaplastic fibrocarcinoma with a very poor

Radiologic and Physiologic Evaluation in Colon and Rectal Surgery; Guest Editor, David W. Dietz, M.D.

Clin Colon Rectal Surg 2008;21:178-187. Copyright (C) 2008 by Thieme Medical Publishers, Inc., 333 Seventh Avenue, New York, NY 10001, USA. Tel: +1(212) 584-4662.

DOI 10.1055/s-2008-1080997. ISSN 1531-0043. 
prognosis. Lymphoma, sarcoma, and squamous cell carcinomas are infrequently seen in the rectum. ${ }^{1}$

Radiology plays a key role in tumor management, from diagnosis and preoperative staging to monitoring of therapy response and postoperative recurrence. Of all the radiologic modalities, magnetic resonance imaging (MRI) provides the best local tumor visualization and soft-tissue contrast. MRI can also characterize complications of rectal cancer, including local invasion, distant metastasis, and postoperative recurrence.

Several treatment options exist for rectal cancer, depending on tumor stage and patient comorbidities. Complete excision is curative, with the surgical approach being determined by tumor location and extent. In general, superficial tumors can be removed by local methods, such as transanal excision or endoscopic microsurgical ablation. High to medium rectal cancers are treated using low anterior resection with total mesorectal excision (TME), in which the rectum is removed en bloc with the complete mesorectal compartment. The minimum tumor distance from the surrounding mesorectal fascia is defined as the circumferential resection margin $(\mathrm{CRM})$, and is an important prognostic factor. Low rectal cancers involve abdominoperineal resection (APR) or coloanal anastomosis. Even more radical operations are required for advanced tumors with close or involved margins. $^{2-5}$

Adjuvant radiation and/or chemotherapy may be utilized for downstaging, which can facilitate total resection or induce complete regression (sterilization); prevention of postoperative local recurrence; and reduction of metastatic risk. Adjuvant regimens are designed with respect to the risk of local recurrence. Superficial tumors, which have a low recurrence risk, can be treated by surgery without adjuvant therapy. Operable tumors with wide resection margins have an intermediate risk for recurrence, and should be given short-course radiation followed by total mesorectal excision. High-risk advanced cancers require a long course of preoperative chemotherapy and radiation, followed by extensive surgery. ${ }^{2-5}$

\section{IMAGING OF RECTAL CANCER}

Current preoperative tests for rectal cancer are frequently inaccurate and unreliable. Fecal occult blood testing (FOBT) is a common screening test for colorectal cancer that is relatively inexpensive and noninvasive, but has low sensitivity and specificity. The same is true of screening using carcinoembryonic antigen (CEA) and other tumor biomarkers. Furthermore, prognostic scoring systems that involve histologic grade and tumor stage (such as the Dukes and AJCC TNM classifications) do not reflect important surgical aspects of disease including local anatomy, circumferential resection planes, and postoperative recurrence (Tables 1 and 2). As a result,
Table 1 Modified Dukes Classification for Rectal Cancer Staging

A Limited to rectal wall

B Extending through rectal wall into extrarectal tissue

B1 Tumor penetration into muscularis propria

B2 Tumor penetration through muscularis propria

C Metastases to regional lymph nodes

C1 Limited to rectal wall with nodal involvement

C2 Penetrating through rectal wall with nodal involvement D Distant metastases (liver, lung, bone)

radiologic imaging is desired for characterization of local tumor extent, surrounding organ involvement, and distant metastasis.

Knowledge of rectal anatomy is key for selecting an optimal imaging modality. The rectum begins at the end of the large intestine, immediately following the sigmoid colon, and ends at the anus. It can be divided into upper (12 to $16 \mathrm{~cm})$, middle ( 6 to $12 \mathrm{~cm}$ ), and lower thirds $(0$ to $6 \mathrm{~cm})$, based on distance from the anocutaneous line. The upper third of the rectum is almost entirely covered by peritoneum. Below this level, the peritoneum reflects anteriorly onto the posterior surface of the female uterus/vagina or male bladder, giving rise to the peritoneal recesses. The lower half of the rectum is completely extraperitoneal. ${ }^{5}$

The rectal wall consists of five distinct layers, namely the mucosa (lined by columnar epithelium), deep mucosa (lamina propria and muscularis mucosae),

Table 2 American Joint Committee on Cancer TNM Classification for Cancers of the Colon and Rectum

Primary Tumor (T)

TX - Primary tumor cannot be assessed or depth of penetration not specified

T0 - No evidence of primary tumor

Tis - Carcinoma in situ (mucosal); intraepithelial or invasion of the lamina propria

T1 - Tumor invades submucosa

T2 - Tumor invades muscularis propria

T3 - Tumor invades through the muscularis propria into the subserosa or into nonperitonealized pericolic or perirectal tissue

T4 - Tumor directly invades other organs or structures and/or perforates the visceral peritoneum

Regional Lymph Nodes (N)

NX - Regional lymph nodes cannot be assessed

NO - No regional lymph node metastasis

N1 - Metastasis in 1-3 pericolic or perirectal lymph nodes

N2 - Metastasis in 4 or more pericolic or perirectal lymph nodes

N3 - Metastasis in any lymph node along the course of a named vascular trunk

Distant Metastasis (M)

MX - Presence of metastasis cannot be assessed

MO - No distant metastasis

M1 - Distant metastasis 
submucosa, muscularis propria (inner circular and outer longitudinal layers), and serosa (perirectal fat). Arterial supply to the rectum includes the superior rectal branch of the inferior mesenteric artery, the middle rectal branch of the internal iliac artery, and the inferior rectal branch of the internal pudendal artery. Venous drainage occurs from the superior rectal to inferior mesenteric vein, middle rectal to internal iliac vein, and inferior rectal to internal pudendal vein. A portosystemic anastomosis is located in the upper anal canal and joins the internal/portal (superior rectal vein) and external/ systemic (middle/inferior rectal veins) venous plexuses. Lymphatic drainage follows the deep vessels, i.e., the upper rectum drains superiorly into the superior rectal nodes; the lower rectum laterally, into the middle rectal nodes; and advanced/anal tumors inferiorly, into the inferior rectal nodes. The mesorectal fascia (fascia propria, perirectal fascia) is a connective tissue sheath that defines the mesorectal compartment. It acts as a primary barrier to tumor spread and contains the rectum, mesorectal fat, blood vessels, lymphatics, and lymph nodes. ${ }^{5}$

Multiple radiologic modalities can be used in the imaging of rectal cancer, each with its unique advantages and limitations. Endorectal/transrectal ultrasound (EUS/TRUS) is used primarily for staging superficial tumors. EUS visualizes the rectal wall layers with high spatial resolution, but suffers from operator dependency, interface reflection artifacts, and a limited depth of acoustic penetration $(<14 \mathrm{~cm})$. Thus, high rectal and bulky/advanced tumors cannot be imaged. Computed tomography $(\mathrm{CT})$, which rapidly images the entire abdomen and pelvis, is used primarily for the assessment of disseminated disease. Accuracy is high for detection of distant metastases, but low for local tumors due to poor spatial/contrast resolution in the region of the rectal wall. Furthermore, CT lacks the true multiplanar capabilities necessary to assess local tumor extent in the appropriate anatomic plane. Positron emission tomography $(\mathrm{PET})$ is used in postoperative evaluation of recurrent rectal cancer, but lacks the spatial resolution necessary for preoperative tumor localization. ${ }^{6,7}$

Overall, MRI is the best technology for evaluating rectal cancer in terms of local extent and recurrence. With the highest soft-tissue contrast of all imaging modalities, MRI is a sensitive and specific tool for tumor staging. Preoperative evaluation of the CRM, TNM (tumor-node-metastasis) stage, and local advancement is possible. Accurate postoperative assessment is also feasible, due to characteristic lesion appearance and signal patterns. ${ }^{6,7}$

\section{MAGNETIC RESONANCE IMAGING TECHNIQUES}

MRI protocols for staging rectal cancer have evolved over the years. Originally, whole-body coils were used and performed similarly to $\mathrm{CT}$, demonstrating reasonable accuracy for distal metastases, but insufficient resolution for lower-stage tumors. The introduction of endoluminal (endorectal) coils provided enhanced spatial resolution. This technique performed comparably to EUS in rectal wall layer evaluation and local lymph node detection. However, imaging was limited to the rectal wall, with marked signal drop-off at the mesorectal fascia and surrounding pelvic structures. Other issues included limited availability, high cost, and difficulties with coil positioning for high/stenosing tumors. The next phase in rectal MRI involved the use of dedicated external coils. Although accuracy was low in early studies, this was largely rectified by the use of newgeneration surface coils. Currently, the favored technology involves phased-array surface coils, which combine high spatial resolution with a sufficiently large field of view. This is less invasive than the endoluminal method, and enables staging of both superficial and advanced tumors.

Techniques for phased-array surface coil MRI of the rectum vary between institutions. Typically, the patient is positioned supine and the phased-array surface coil centered on the pelvis, with lower edge well below the pubic bone. No special patient preparation is required. Rectal cleansing is controversial: although air insufflation and contrast administration reduce artifact from stool residue, the resulting luminal distention may affect evaluation of the CRM. Intramuscular or intravenous spasmolytics can also be administered to avoid peristaltic artifacts. Ideally, the bladder should be moderately full to prevent motion artifact. The total duration of the study ranges from 25 to 45 minutes.

For planning purposes, a T2-weighted breathhold sequence with a large field of view (FOV) is rapidly acquired in the sagittal plane, and optionally the axial and coronal planes. Sequences can involve single-shot HASTE (single-shot fast spin echo [SSFSE], halfFourier acquired single turbo spin-echo [HASTE], single-shot turbo spin echo [SSTSE]), or balanced gradient echo (fast imaging employing steady-state acquisition [FIESTA], true fast imaging with steadystate precession [TrueFISP], balanced fast field echo [BFFE]).

Localization scans are then performed using an axial T2-weighted fast spin-echo (FSE) or turbo spin-echo (TSE) sequence with a large FOV and slice thickness. When performing the diagnostic examination, scan planes should be precisely aligned relative to the longitudinal axis of the tumor or surrounding intestinal lumen. This enables accurate relation of the tumor to the surrounding intestinal wall, mesorectal fascia, extramural veins, lymph nodes, peritoneal folds, anal sphincter, and pelvic organs.

The crux of the examination involves a high-resolution T2-weighted sequence, generally a 


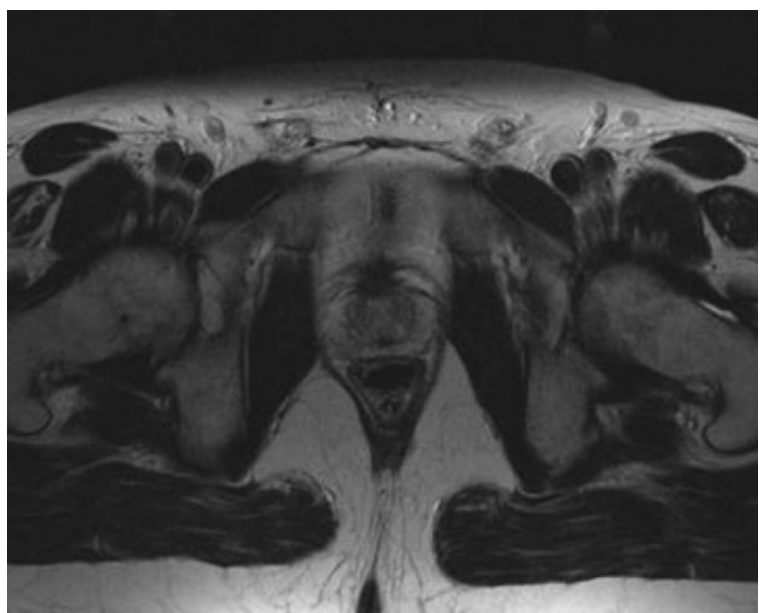

Figure 1 Rectal wall layers. Three rectal wall layers can be distinguished on magnetic resonance imaging: an inner hyperintense layer, representing the mucosa and submucosa; an intermediate hypointense layer, the muscularis propria; and an outer hyperintense layer, the serosa (perirectal fat).

non-breath-hold FSE/TSE. A high-resolution matrix, thin collimation, and small FOV improve in-plane spatial resolution. With this technique, fat tissue remains high in signal intensity, and provides excellent contrast against hypointense structures such as tumor and mesorectal fascia. Three rectal wall layers can be distinguished on MRI: an inner hyperintense layer, representing the mucosa and submucosa; an intermediate hypointense layer, the muscularis propria; and an outer hyperintense layer, the serosa (perirectal fat). The mesorectal fascia is identified as a hypointense linear structure surrounding the mesorectum and perirectal fat (Fig. 1).

Images are acquired in the transverse, coronal, and sagittal planes to characterize rectal wall anatomy with regard to tumor length and complex spatial relationships. Axial images are used to localize tumor mass and extent. Sagittal images enable assessment of the sacrum, bladder, uterus, cervix, vagina, prostate, and thecal sac. Coronal images are important for evaluating the mesorectal fascia, vessels, pelvic sidewall/floor, and anal canal.

Optional T1 to proton density-weighted images can be obtained through the entire pelvis. These can involve two-dimensional (2D) FSE/TSE sequences with short echo train lengths or three-dimensional (3D) gradient-echo sequences. T1-weighted images aid in assessment of tumor invasion and distant lymphadenopathy. ${ }^{8,9}$

Fat suppression techniques and contrast administration may improve visualization, but are not usually required. Studies using paramagnetic contrast agents have not demonstrated significant improvements in preoperative staging. However, contrast should be given whenever T1-weighted images are acquired, and for postoperative assessment. ${ }^{10}$

\section{CIRCUMFERENTIAL RESECTION MARGIN}

The CRM for TME is defined as the minimum distance from tumor to mesorectal fascia, and determines the surgical resection plane necessary to achieve tumor-free margins. The CRM is the most powerful predictor of local recurrence rate. It can be used to identify tumors with close/involved resection margins, thus requiring neoadjuvant treatment and more aggressive surgery.

Preoperative MRI prediction of the CRM has demonstrated extremely high accuracy and precision in histopathologic studies. The CRM is said to be involved when tumor is seen within $1 \mathrm{~mm}$ of the mesorectal fascia, or has visibly invaded it. A tumor-free CRM is assumed when the closest visible tumor extension, mesorectal tumor deposit, or suspicious lymph node is over $6 \mathrm{~mm}$ from the mesorectal fascia. There is no consensus on the status of tumors located 2 to $5 \mathrm{~mm}$ from the mesorectal fascia (Fig. 2). ${ }^{11-13}$

\section{T STAGE}

Endorectal MRI has an accuracy comparable to EUS for $T$ staging of superficial tumors. However, the small field of view limits evaluation of advanced cancers. Phasedarray MRI is suitable for staging of both superficial and advanced tumors, but accuracy is only slightly better than full-body coils or CT. This is largely a result of the inability to differentiate $\mathrm{T} 1$ to $\mathrm{T} 2$ cancers and borderline T3 cancers. Overstaging is caused by fibrotic desmoplastic reactions, which mimic true mesorectal tumor invasion. These issues are not unique to MRI, but are also seen in other imaging modalities (CT, EUS) (Fig. 3).

However, the T-staging system is of questionable clinical significance. In the current system, a T3 tumor may be well circumscribed, with a wide resection margin; or bulky and invasive, with a narrow margin. Yet the latter tumor has a much higher risk of recurrence, and should be managed differently. The CRM is a much more accurate predictor of local recurrence rate for such tumors, and is excellently described by MRI. Infiltration of extramural veins and the peritoneal fold are additional prognostic factors that can be identified on MRI (Fig. 4). ${ }^{11-13}$

\section{N STAGE}

$\mathrm{N}$ stage is a prognostic indicator for both distant metastases and local recurrence. Thus, determination of nodal status is key in planning surgical and adjuvant therapy. With standard TME, the mesorectum and perirectal 
A
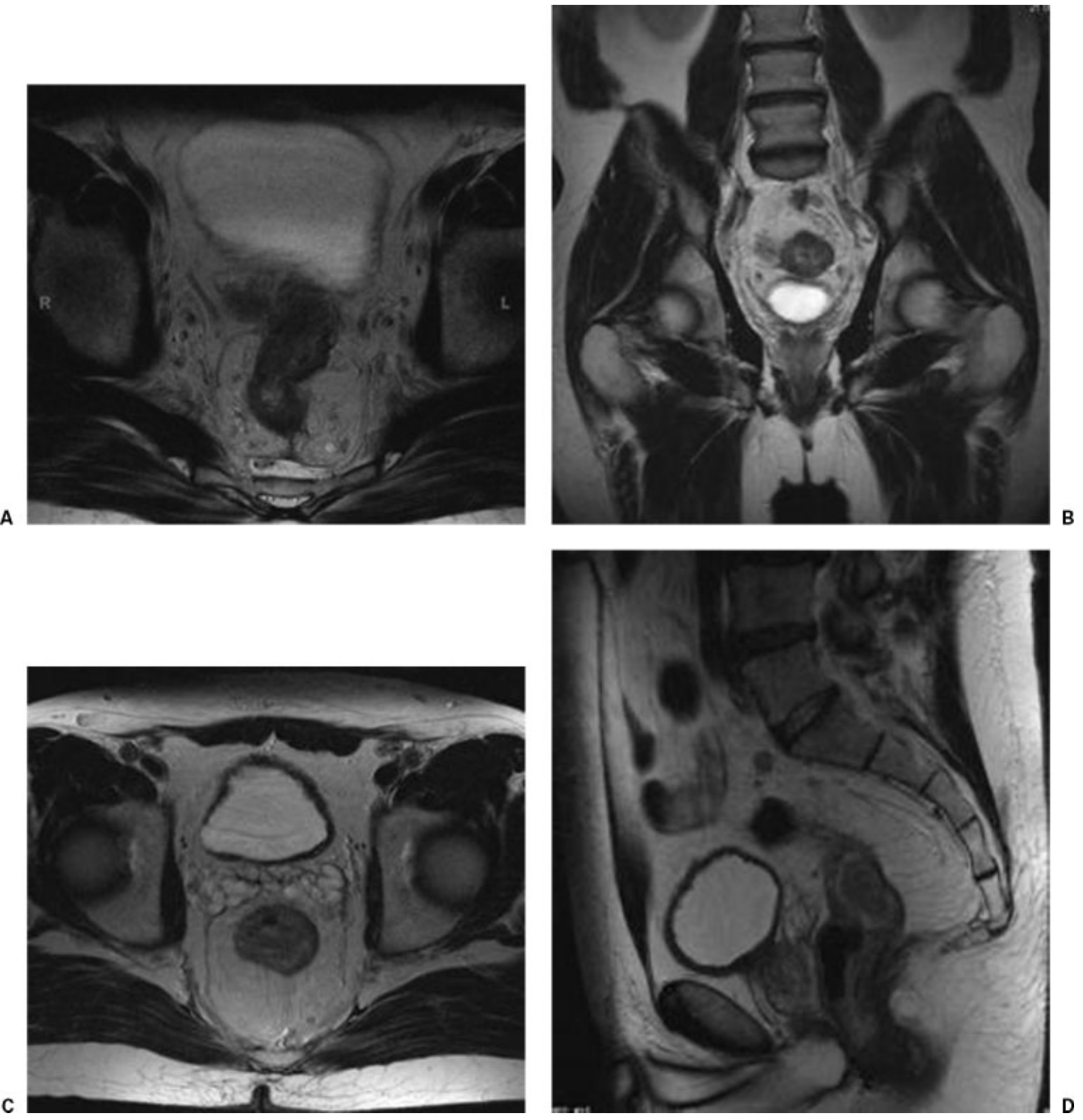

Figure 2 Involved circumferential resection margin (CRM). (A) Axial. (B) Coronal. The CRM is said to be involved if tumor is within $1 \mathrm{~mm}$ of the mesorectal fascia or has visibly invaded it. Tumor-free CRM. (C) Axial. (D) Sagittal. A tumor-free CRM is assumed when the closest tumor extension, mesorectal tumor deposit, or suspicious lymph node is over $6 \mathrm{~mm}$ from the mesorectal fascia.

nodes are removed, but the internal iliac nodes are left in situ. In lower rectal cancer, and when nodes outside the mesorectum are seen, wider resection with removal of the internal iliac nodes becomes necessary. Some surgeons employ a highly aggressive approach with extended pelvic lymphadenectomy, but the benefits of this are unclear.

Unfortunately, identification of metastatic lymph nodes is the most challenging issue in rectal cancer imaging. Assessment is based on nonspecific morphologic criteria such as size and shape. Using a size cutoff
$(>3,5,10 \mathrm{~mm})$ identifies abnormally enlarged nodes, but does not distinguish metastatic versus benign inflammatory etiologies. Moreover, such a criterion will exclude micrometastases to normal-sized lymph nodes, which are common in rectal cancer. To date, the most accurate criteria for suspicious lymph nodes on MRI are irregular contour (spiculated or indistinct borders) and heterogeneous signal (mottled intensity pattern). There are no known signal intensity/enhancement properties that can reliably distinguish metastatic from reactive nodes. ${ }^{11-13}$ 

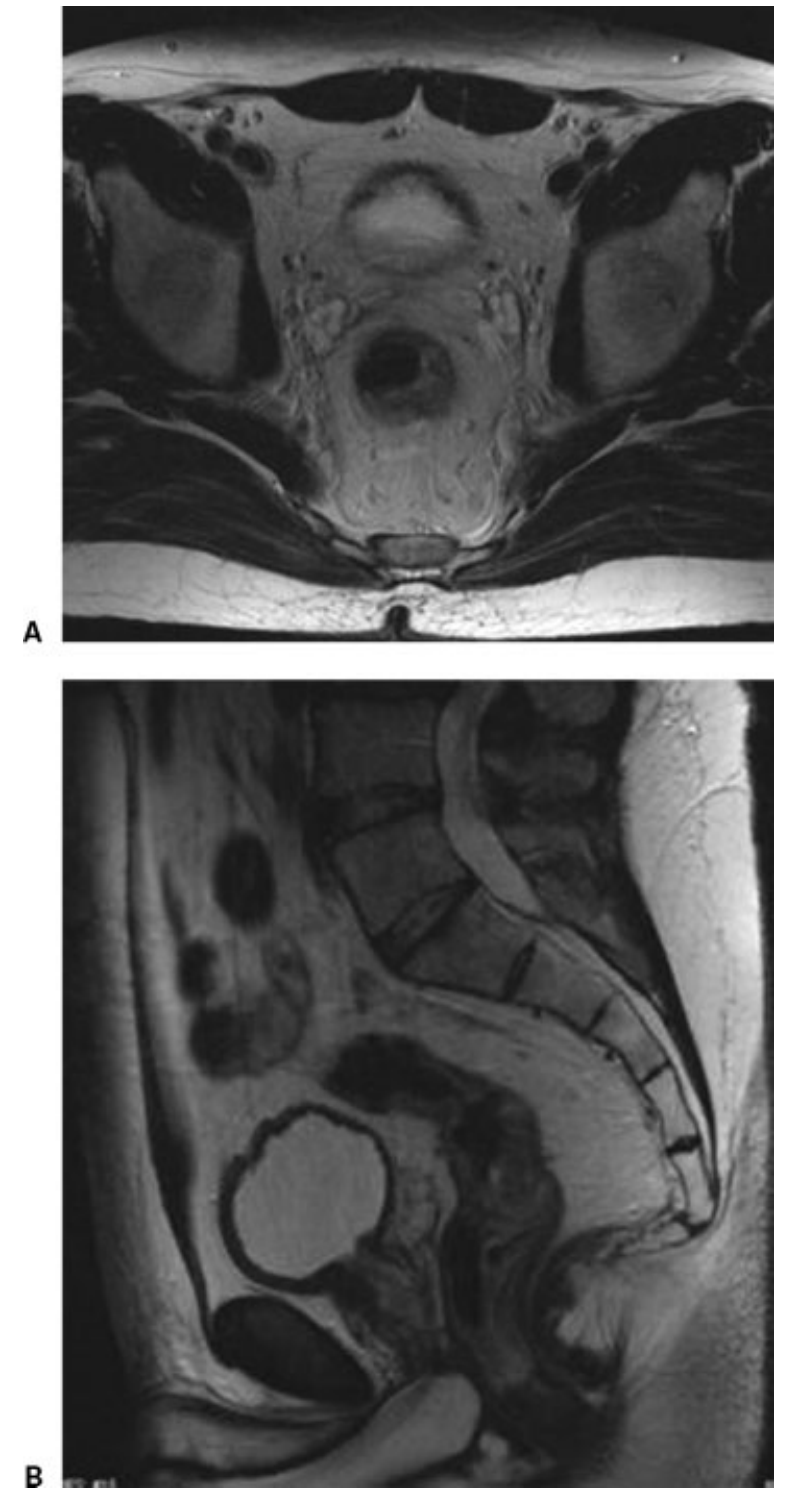

Figure 3 Overstaging of T2 rectal cancer. (A) Axial. (B) Sagittal. Desmoplastic reaction of tumor into adjacent perirectal fat.

\section{LOCALLY INVASIVE CANCER}

Locally advanced rectal cancer with fixation to surrounding pelvic structures should be treated with extensive neoadjuvant therapy, followed by radical en bloc resection. Superior anatomic visualization is necessary for assessing tumor extent and surgical planning. MRI is preferred over CT, due to its multiplanar capabilities and superior soft-tissue contrast. On MRI, fat planes are easily visualized along with invasion into the pelvic floor/ anal canal, pelvic sidewall, bladder, uterus/cervix/vagina, prostate, spinal cord, and bone marrow. Complications of organ invasion include bowel obstruction/perforation and fistulization to the small bowel, bladder, and vagina (Fig. 5). ${ }^{11-13}$

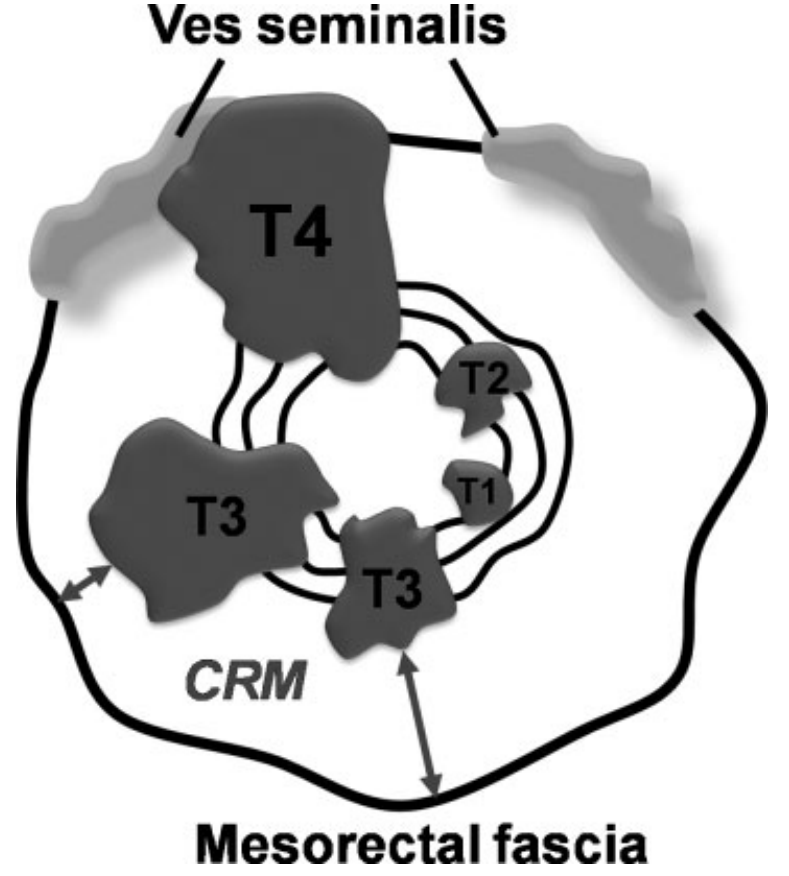

Figure 4 Schematic of circumferential resection margin (CRM) versus T stage. The current T-staging system does not distinguish tumors with wide versus close/involved resection margins. The CRM measurement is of far more clinical significance. Short tumor-mesorectal fascia distances alter surgical planning and may require neoadjuvant treatment, due to the increased local recurrence risk.

\section{STAGE}

Rectal cancer, like colon cancer, usually metastasizes to the liver. This is a result of lymphatic drainage from the superior rectal vessels into the superior/inferior mesenteric veins and portal venous system. Abdominal MRI is the preferred modality for detecting liver metastases, particularly when volume calculations are needed for resection planning. Pulmonary metastasis can also occur as a result of lower rectal drainage into the internal iliac veins and systemic venous system. Additional sites of spread include the adrenals, ovaries, retroperitoneum, and omentum. Bone and cerebral metastases are uncommon (Fig. 6). ${ }^{11-13}$

\section{POSTOPERATIVE ASSESSMENT}

Local recurrence of rectal cancer is usually due to incomplete surgical excision, and is commonly predicted by CRM invasion. Other risk factors include tumor stage and grade, distance from the anal verge, presence of lymphovascular invasion, anastomotic leakage, and tumor perforation during resection. In certain cases, complete resection can prolong survival. However, surgical risks may be unacceptably high for advanced tumors requiring radical operations (partial sacrectomy, pelvic exenteration). Absolute contraindications to resection include involvement of the S1 to S2 nerve roots, proximal 
A
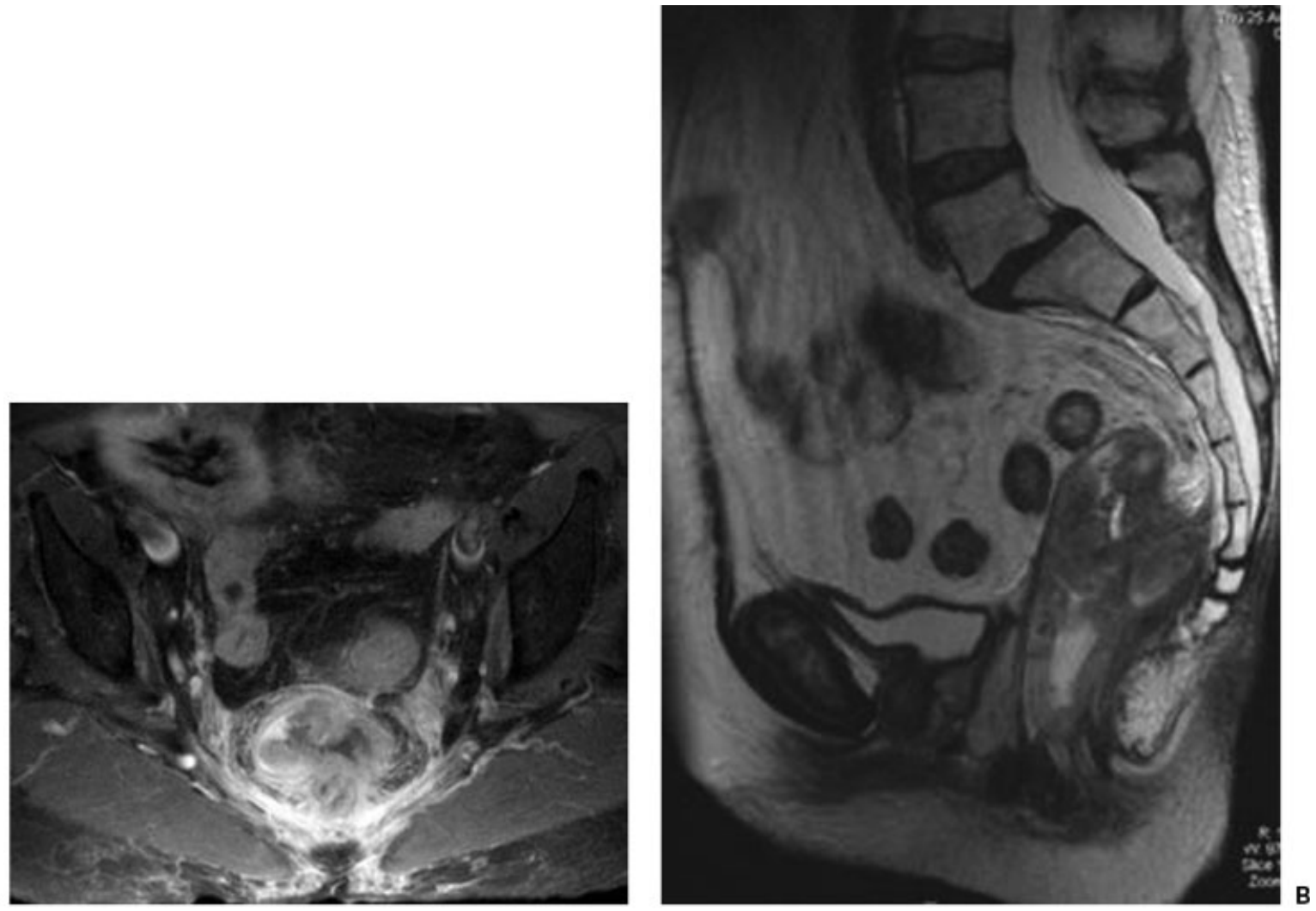

B
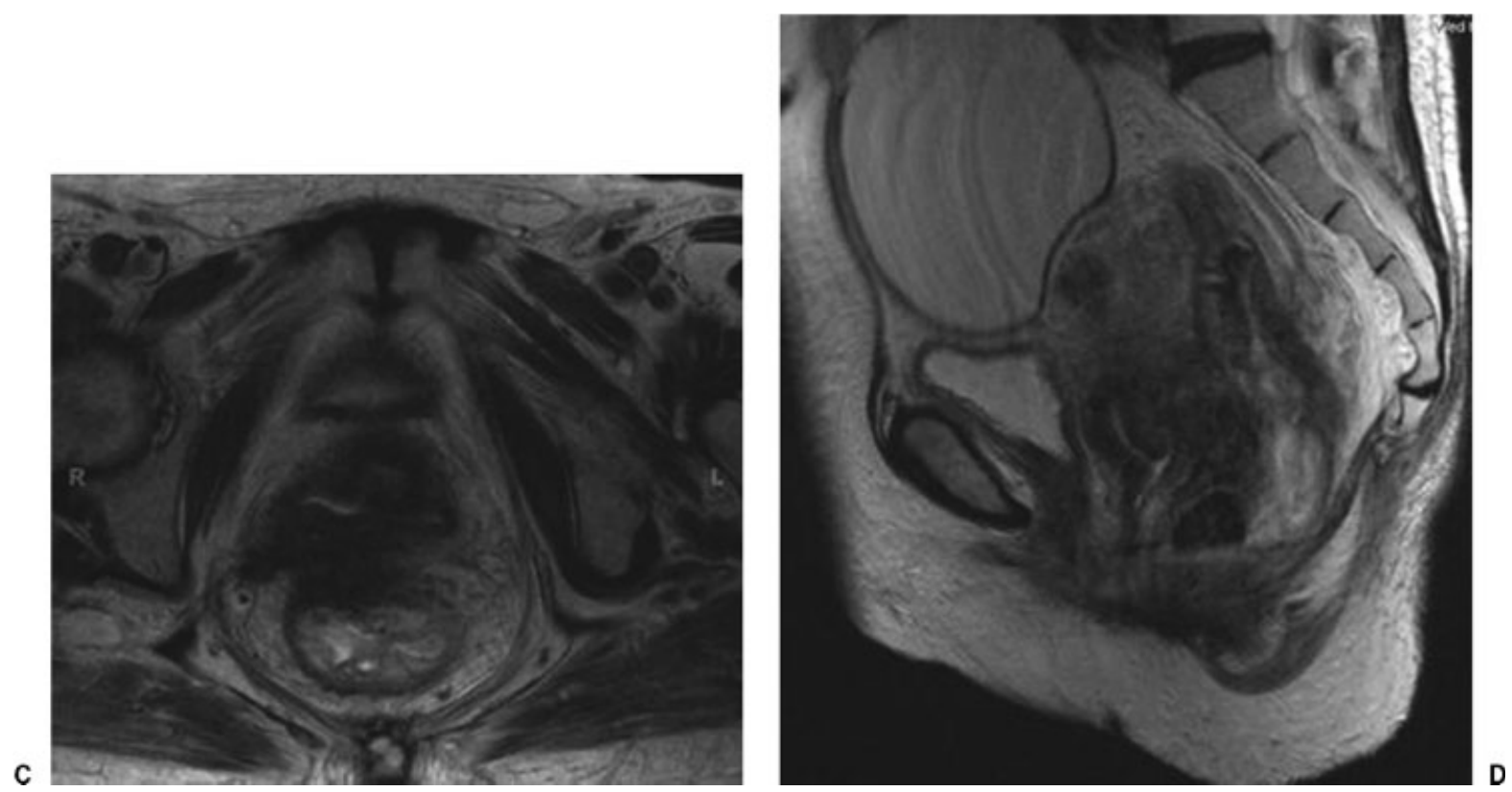

Figure 5 Infiltration of pelvic sidewall. (A) Axial. (B) Sagittal. Invasion into uterus/cervix/vagina. (C) Axial. (D) Sagittal.

sacral invasion above the level of the S2 to S3 junction, extensive pelvic sidewall involvement with encasement of the iliac vessels, and extension through the greater sciatic notch. Limited pulmonary and hepatic metastases are often resectable, and do not preclude surgery for recurrent pelvic tumors.

Serial imaging scans can be used to monitor patients for tumor recurrence following rectal cancer surgery. Local recurrence is often seen at the resection margins, which may be abdominoperineal, low anterior, or superficial depending on the type of surgery performed. Tumors can also arise at the rectal wall, and may be extraluminal or intraluminal. Intraluminal recurrence is less common and usually occurs at the anastomotic line; this is best visualized by endorectal MRI or other endoscopic techniques. Cancers may also 
A

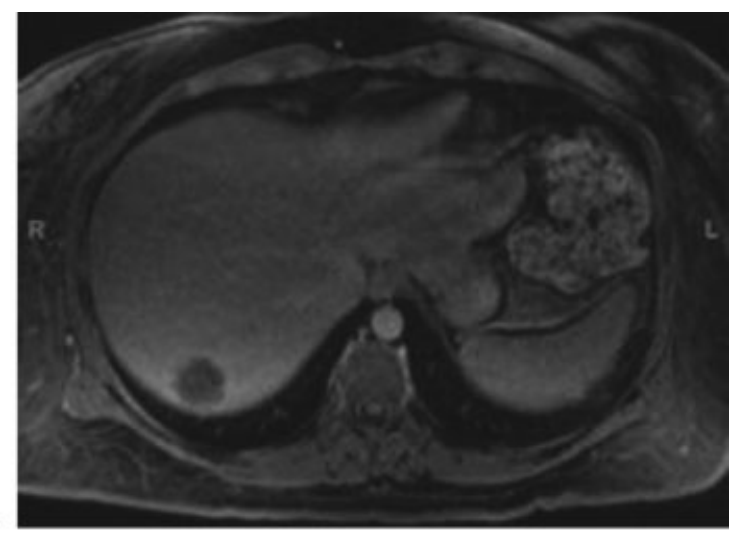

B

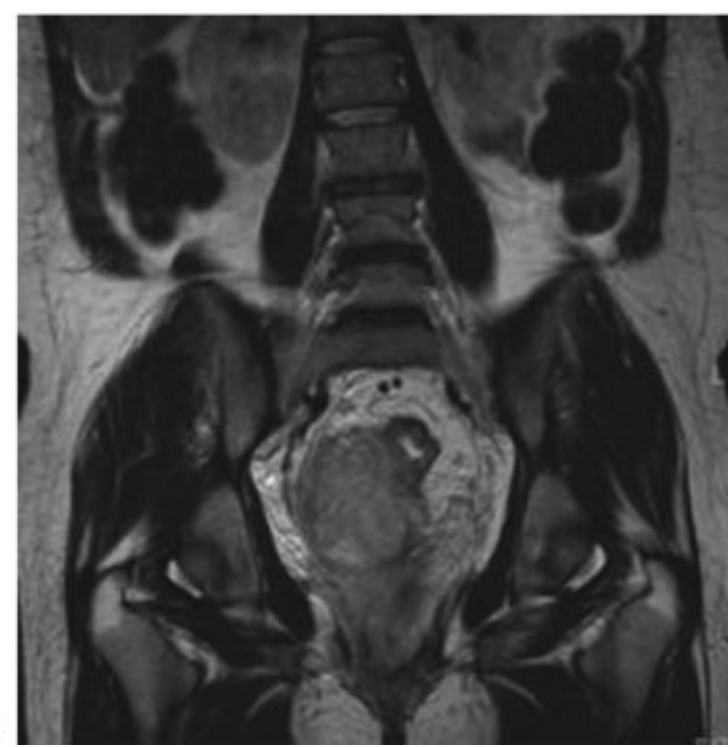

Figure 6 Liver metastases from rectal cancer. (A) Axial image demonstrates liver lesion. (B) Coronal image shows extent of rectal cancer.

develop in the pelvic organs and are classified by location (central, pelvic sidewall, or sacral). Central recurrence (bladder, prostate, seminal vesicles, uterus, vagina, or small bowel) is the most common and has the best prognosis. The sacrum is the least common site of recurrence and carries an intermediate survival rate. Pelvic sidewall recurrence harbors the worst prognosis.

MRI is the most sensitive and specific imaging modality for detecting tumor recurrence. Appearances vary with the histology of the primary tumor, but lesions are usually intermediate to high intensity on T2weighted images and show marked contrast enhancement. However, if a large fibrous component is present, tumors may display low signal intensity and little to no enhancement. This is particularly common with small/ early lesions and after therapeutic complications such as anastomotic leak or hemorrhage.

It can be difficult to distinguish recurrent tumors from postsurgical/radiation changes. Morphologically, recurrent cancer often presents as an irregular globular mass that increases in size or changes shape over time. Central necrosis may be present. In contrast, postsurgical inflammation produces soft tissue thickening with adjacent fat stranding, which resolves over time. Radiation produces early bowel wall thickening, edema, and ulceration. Late changes include stricture/fistula formation, fatty bone marrow replacement, and adjacent muscle atrophy.

On MRI, mature fibrosis is readily differentiated from recurrent tumor, based on its low T1-/T2weighted signal intensity and lack of enhancement. However, acute/subacute tissue reactions may be indistinguishable from tumor, and persist for up to 12 months following surgery. Granulation tissue, hematoma, and radiation-induced inflammation may all produce mural thickening, high T2-weighted signal intensity, and contrast enhancement (Fig. 7).

Some authors suggest that the shape of a mass can help distinguish tumor from fibrosis. Recurrence is favored when the lesion has a rounded nodular appearance, whereas straight angular margins are more suggestive of fibrosis. The combination of this criterion with high T2-weighted signal intensity and contrast enhancement over $40 \%$ is highly sensitive and specific for local recurrence. Another criterion involves the
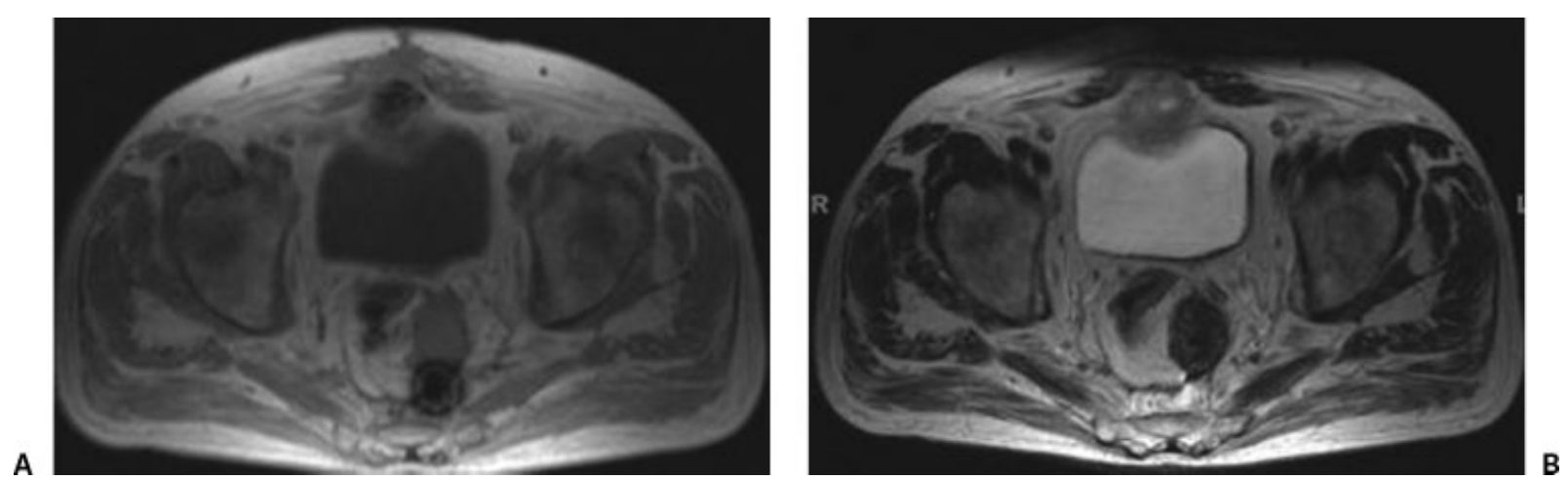

Figure 7 Giant cell reaction to foreign material. (A) T1-weighted magnetic resonance imaging (MRI). (B) T2-weighted MRI. Mature fibrosis is readily differentiated from recurrent tumor based on a low T1-/T2-weighted signal intensity and lack of gadolinium enhancement. 
A

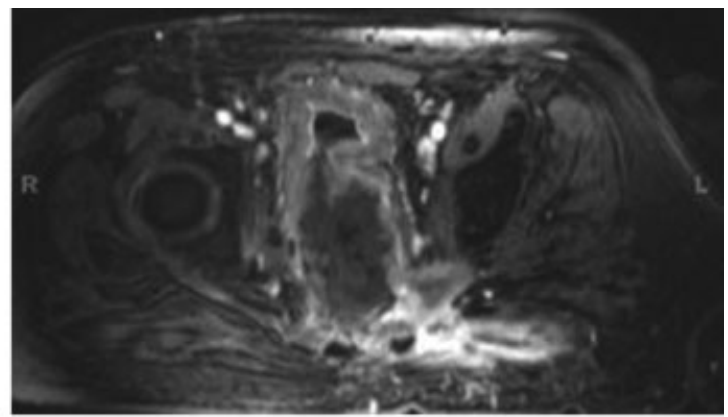

B

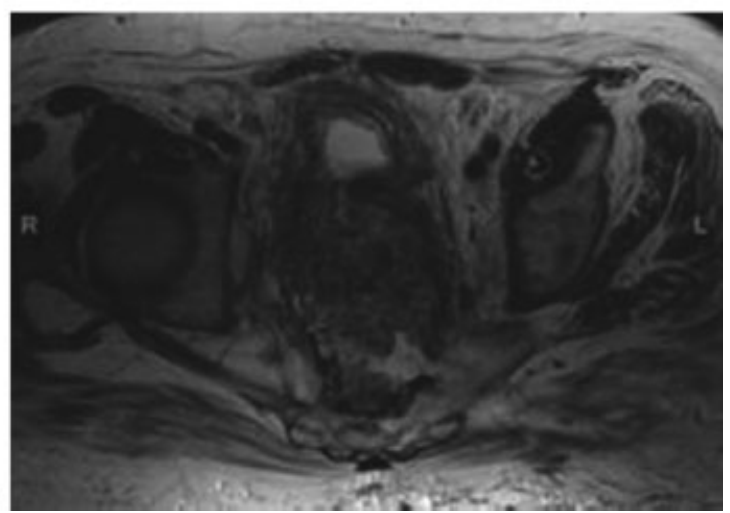

Figure 8 Rim-enhancement pattern. (A) T1-weighted magnetic resonance imaging (MRI). (B) T2-weighted MRI. Recurrent tumor displays a central hypointense area surrounded by a strongly enhancing margin of variable thickness. Postsurgical abscesses may show a similar appearance. Enhancement can also be seen with early fibrosis, but the rim-enhancement pattern has not been described.

"rim-enhancement pattern," in which recurrent tumor displays a central hypointense area surrounded by a strongly enhancing margin of variable thickness. Postsurgical abscesses may show a similar appearance. Enhancement can be seen with early fibrosis, but the rim-enhancement pattern has not been described (Fig. 8).

Another issue involves differentiation of tumor invasion from normal anatomic contiguity. In the postsurgical or irradiated pelvis, fat planes are often grossly distorted or absent and cannot reliably predict invasion. Definite involvement is assumed when tumor tissue visibly invades or destroys adjacent anatomy, signal changes in adjacent tissue reflect those of the recurrent tumor, or muscle enlargement is identified. The latter criterion applies 12 or more months following surgery, at which point other causes of muscle enlargement such as hematoma and inflammation have resolved. ${ }^{14-16}$

\section{CONCLUSIONS AND FUTURE DIRECTIONS}

Considerable advances have been made in the diagnosis and treatment of rectal cancer over the last decade. Currently, MRI is the only imaging modality that enables prediction of the CRM, as well as preoperative local staging and postoperative assessment. This has helped greatly in determining patient prognosis and planning of surgical/adjuvant therapy.

Currently, endorectal MRI provides the best visualization of rectal wall layers for superficial rectal cancers, whereas phased-array MRI is preferred for evaluating the mesorectal fascia and CRM in locally advanced cancers. However, MRI is a rapidly advancing field, and imaging techniques for rectal cancer will need to be continually optimized. Future research in MRI is geared toward accessory technologies such as highresolution MRI, whole-body scans, and parallel imaging; imaging of lymph nodes by MR lymphography; and response to therapy using diffusion/perfusion-weighted MR and functional imaging.

High-resolution (thin-section) MRI using multielement wraparound surface coils enables visualization of all five rectal wall layers, leading to more accurate determination of the CRM and local tumor extent. ${ }^{17}$ The advent of whole-body MRI systems enables imaging of the entire body in a single session via repeated table movement. Studies have demonstrated a multitude of diagnostic applications in oncologic patients, and may be used for distal staging of rectal cancer in the future. Parallel imaging techniques have also been used to shorten MRI examination times without compromising diagnostic accuracy.

There is evidence that MRI is superior to conventional CT for assessing local and pelvic involvement in rectal cancer. The new-generation multidetector row spiral CT (MDCT) scanners have not been fully explored, but offer good contrast/spatial resolution with fast scan times and relatively low cost. Further studies are needed to examine the roles of full-body MRI in distal cancer spread and MDCT in local tumor extension. If either of these investigations succeed, it will become possible to combine local, regional, and distal staging in a single examination.

As previously discussed, imaging of lymph nodes is the greatest challenge in preoperative rectal cancer imaging. Positron emission tomography (PET) has been used for nodal staging with disappointing results, largely due to overlying bladder artifacts. MR lymphography is a novel experimental approach that utilizes ultrasmall superparamagnetic iron oxide (USPIO) contrast agents. Following intravenous or rectal administration, USPIO particles are selectively taken up by reticuloendothelial macrophages in normal lymph nodes. These molecules shorten T2-/T2*-weighted relaxation times, inducing a signal decrease on proton density- and T2-weighted images. In pathologic nodes, the reticuloendothelial system is displaced by neoplastic cells. Thus, decreased USPIO uptake produces a relatively bright signal intensity. MR lymphography has promising applications in the detection of tumor micrometastases. Initial results for double-contrast imaging with gadolinium and rectal 
USPIO demonstrate high accuracy in the detection of mesorectal lymph nodes.

For follow-up imaging, monitoring of therapeutic response and postoperative recurrence are key objectives. Diffusion- and perfusion-weighted MRI are new imaging techniques that focus on the microscopic properties of tumor tissue, rather than their macroscopic extent. Diffusion-weighted MRI reflects the translational motion of water molecules, which is closely related to biological tissue changes. Characteristic changes in the apparent diffusion coefficient (ADC) have been reported in the progression from interstitial edema to cytotoxic edema and end-stage fibrosis/necrosis.

Perfusion-weighted or dynamic contrastenhanced MRI (DCE-MRI) is based on the assumption that tumor enhances earlier and to a greater extent than normal tissue, due to its increased regional blood flow and vascular permeability. Both low-molecular-weight and macromolecular (blood pool) contrast agents have been used with varying results. A kinetic model is used to calculate tumor perfusion parameters such as blood flow, volume, capillary permeability, and transit time. This is a computationally intensive, but promising technology for assessing tumor angiogenesis, which is essential for tumor growth and metastasis. Studies have shown that treatment response is positively correlated with increased tumor vascularity, likely due to increased tumor dependence on oxygenation and improved drug delivery. ${ }^{18,19}$

Functional imaging, which provides information regarding the biological behavior of tumors, has the potential to augment information gained from crosssectional studies. For example, positron emission tomography (PET) can identify malignant cells via increased concentration of the radioactive glucose analog 18fluorodeoxyglucose (FDG). This can help identify residual or recurrent disease, as well as predict tumor response to adjuvant therapy. However, the spatial resolution of PET is low, and false-positives may occur with inflammation and postsurgical/radiation change. Another functional approach is immunoscintigraphy, which uses radiolabeled monoclonal antibodies targeting CEA. Due to its high specificity but low sensitivity/ accuracy, this is used as a second-line test in the detection of local recurrence. In the future, combined nuclear medicine/radiologic studies such as PET-MRI will effectively unite the best aspects of metabolic and anatomic imaging.

\section{REFERENCES}

1. Hussain SM, Outwater EK, Siegelman ES. Mucinous versus nonmucinous rectal carcinomas: differentiation with MR imaging. Radiology 1999;213(1):79-85
2. Beets-Tan RG, Beets GL. Rectal cancer: review with emphasis on MR imaging. Radiology 2004;232(2):335346

3. Klessen C, Rogalla P, Taupitz M. Local staging of rectal cancer: the current role of MRI. Eur J Radiol 2007;17(2): 379-389

4. Iafrate F, Laghi A, Paolantonio P, et al. Preoperative staging of rectal cancer with MR imaging: correlation with surgical and histopathologic findings. Radiographics 2006;26(3):701714

5. Lahaye MJ, Lamers WH, Beets GL, Beets-Tan RGH. MR Anatomy of the rectum and the mesorectum. In: Di Falco G, Santoro GA, eds. Benign Anorectal Diseases: Diagnosis with Endoanal and Endorectal Ultrasound and New Treatment Options. New York, NY: Springer; 2006:67-77

6. Sahani DV, Kalva SP, Hahn PF. Imaging of rectal cancer. Semin Radiat Oncol 2003;13(4):389-402

7. Dobos N, Rubesin SE. Radiologic imaging modalities in the diagnosis and management of colorectal cancer. Hematol Oncol Clin North Am 2002;16(4):875-895

8. Vliegen RF, Beets GL, von Meyenfeldt MF, et al. Rectal cancer: MR imaging in local staging-is gadolinium-based contrast material helpful? Radiology 2005;234(1):179188

9. Wallengren NO, Holtas S, Andren-Sandberg A, Jonsson E, Kristoffersson DT, McGill S. Rectal carcinoma: doublecontrast MR imaging for preoperative staging. Radiology 2000;215(1):108-114

10. Elmas N, Killi RM, Sever A, Klessen C, Rogalla P, Taupitz $\mathrm{M}$. Colorectal carcinoma: radiological diagnosis and staging. Eur J Radiol 2002;42(3):206-223

11. Goh V, Halligan S, Bartram CI. Local radiological staging of rectal cancer. Clin Radiol 2004;59(3):215-226

12. Maier A, Fuchsjager M. Preoperative staging of rectal cancer. Eur J Radiol 2003;47(2):89-97

13. Moran B, Brown G, Cunningham D, Daniels I, Heald R, Quirke P, Sebag-Montefiore D. Clarifying the TNM staging of rectal cancer in the context of modern imaging and neoadjuvant treatment: ' $y$ ' 'u' and 'p' need 'mr' and 'ct'. Colorectal Dis 2008;10(3):242-243

14. Hoeffel C, Arrivé L, Mourra N, Azizi L, Lewin M, Tubiana JM. Anatomic and pathologic findings at external phasedarray pelvic MR imaging after surgery for anorectal disease. Radiographics 2006;26(5):1391-1407

15. Messiou C, Chalmers A, Boyle K, Sagar P. Surgery for recurrent rectal carcinoma: The role of preoperative magnetic resonance imaging. Clin Radiol 2006;61(3):250258

16. Tan PL, Chan CL, Moore NR. Radiological appearances in the pelvis following rectal cancer surgery. Clin Radiol 2005; 60(8):846-855

17. Brown G, Richards CJ, Newcombe RG, et al. Rectal carcinoma: thin-section MR imaging for staging in 28 patients. Radiology 1999;211(1):215-222

18. Barrett T, Kobayashi H, Brechbiel M, Choyke PL. Macromolecular MRI contrast agents for imaging tumor angiogenesis. Eur J Radiol 2006;60(3):353-366

19. Zahra MA, Hollingsworth KG, Sala E, Lomas DJ, Tan LT. Dynamic contrast-enhanced MRI as a predictor of tumour response to radiotherapy. Lancet Oncol 2007;8(1):6374 